\title{
Advanced mathematical model of transfer and diffusion process of harmful substances in the atmospheric boundary layer
}

\author{
Normanmad Ravshanov, Daler Sharipov \\ Centre for the development of software and hardware-program complexes at Tashkent University of \\ Information Technologies, laboratory Modeling of Complex Systems, Uzbekistan \\ ravshanzade-09@mail.ru, Qushkor@mail.ru
}

\begin{abstract}
In the paper, we describe a mathematical model of the spread of harmful substances in the atmospheric boundary layer, taking into account the terrain and the characteristics of the underlying surface, described by means of a system of differential equations in partial derivatives and corresponding initial and boundary conditions. The equations of motion of multicomponent air, equations for calculating the pressure and heat flow for gas and condensate are used in the model.

To determine the velocities of the air mass in the atmosphere in three directions $u, v$ and $w$ we consider the hydrodynamic equations of Navier-Stokes, and to compute the density of substances emitted into the atmosphere, taking into account the law of conservation of mass to the fluid flow through the fixed volume we use mass continuity equation.

To calculate the heat transfer in a multicomponent environment in the transfer of heat to the gas, and to determine the vapor pressure in terms of temperature, we use the equation of Mendeleev-Clapeyron.

The equations are given for describing the transition of water from liquid to gaseous state and vice versa, and when gas, water vapor, liquid water and soot are thrown from the source.
\end{abstract}

Keywords: mathematical model, differential equations, the terrain, the process of pollution, multicomponent environment, ecology, transfer and diffusion of harmful substances.

\section{I.Introduction}

The rapid development of industries, production and development of oil, gas and mineral deposits, processing of raw materials and products for general purpose, building production facilities and residential community has set mankind acute problem of the environment. As a result of a sharp rise in noxious emissions in the industrial regions the concentration of harmful substances in the atmosphere exceeded the maximum allowable health norms. The problems associated with the extraction of coal, nonferrous metals and other minerals have led to soil erosion and the contamination of large areas of secondary materials and waste products that serve as a source of pollution of the waters of cities and regions.

It also should be noted that gas impurities emitted from thermal power plants, factories and production facilities undergo complex chemical reactions, which arise as a result of new, more toxic substances that were not in the original releases. These include inorganic substances particularly harmful emissions of nitrogen oxides and sulfur oxides and carbon dioxide, etc. As we know, all the harmful substances thrown out from industrial production facilities in the environment (surface layer of the atmosphere), ultimately as a material substance deposited on the surface of the earth, and heavy ones precipitate mainly by the gravitational field, and light ones as a result of the diffusion process.

The growth of human impact on the environment caused by the intensive use of natural resources, production of energy from the earth, as well as the development of material production, has led to the disruption of the ecological balance locally - in some parts of the world, and globally - on the planet as a whole. This is particularly noticeable in countries with the rapid growth of the productive capacity of the production facilities and primary processing of raw materials, as an example in the countries of India, China, Korea, Singapore and etc.

As a result of violation of the ecological balance in the globe cancer, asthma, allergies and other diseases began to rise sharply, declines have been occurring in many species of fauna and flora.

It is necessary to emphasize that for research, forecasting and monitoring of the atmospheric pool of industrial regions, as well as to estimate the impact of anthropogenic factors it is necessary to develop a tool which can be used to solve the above problems. One of the most effective and constructive methods and tools for the task is - mathematical modeling and computational experiments, which we can give a qualitative and quantitative assessment of the environmental situation of any region. 
To solve these urgent problems of global scale scientific research centers and schools are established under the guidance of leading specialists in various branches of science dealing with problems of environmental protection, protection of water resources from external technological factors, the impact of the transformations in the ecosystem. Significant results with theoretical and applied character are obtained by scientific schools and centers recently. The literature review shows the list of urgent problems, which are being solved with the help of mathematical modeling occupy a special place.

In the work [1-3], the authors developed a kinematic model of the distribution of reactant particles in the cloud described by the system of quasi-linear partial differential equations of parabolic type, axisymmetric jets, typical cumulonimbus cloud, which considers the transfer processes, diffusion, generation and dissipation on the development of turbulence and investigated the behavior of the approximate solution, depending on the selected grid.

In [4], the modeling problem of the dispersion of hazardous gaseous emissions in the atmosphere is discussed and marked by three main approaches for modeling this process: Gaussian dispersion model called dispersion models; the models, based on the integral conservation laws of substances; models, based on numerical solution of the conservation equations - numerical simulation. The mathematical model, developed in this paper, describes the following processes: cloud movement at variable speed of vertical wind; gravitational spreading; scattering of cloud in vertical direction by atmospheric turbulence; heating or cooling of cloud due to air mixing; heat exchange between clouds and the underlying surface. The authors of the study took into account the changes in the mass and internal energy of the cloud and its physical characteristics in the process of transfer and diffusion of harmful particles in the atmosphere and compared the results of model calculations with experimental data. As the result of the numerical calculations they made the following conclusions: standard procedures based on Gaussian models are not able to reliably predict the spread of harmful substances (heavy gases) as a once only and a constant source of harmful emissions into the atmosphere.

The article [5] presents the main approaches to the creation of computer models of atmospheric phenomena. A review of modern models of distribution of the substance in the atmosphere, filter dust and pollen and the advantages of the model SILAM Finnish Meteorological Institute are presented. The physical side of the problem is related to the analysis of the issue; distribution and absorption of pollutants are considered in the work.

In the work [6] the significant factors affecting the process of transfer and diffusion of harmful substances: the regime of atmospheric circulation, its thermal stability; atmospheric pressure, humidity, temperature control; temperature inversions, their frequency and duration; wind speed, repeatability of air stagnation and weak wind (speed of $1 \mathrm{~m} / \mathrm{s}$ ); duration of fog; terrain, geological structure and hydrogeology of the area; soil and vegetation conditions (soil type, water permeability, porosity, particle size distribution of the soil, the state of the vegetation, species composition, age, site class); background values of pollution of natural components of the atmosphere; condition of the animal world are shown.

In [7] a distributed automated system is developed with the use of the modern information technology, which allows the increase in efficiency of research and forecasting the spread of pollutants emitted by chemicaltechnological enterprises in the industrial atmosphere of region.

The works [8-9] are devoted to a critical analysis of the applicability of physical and mathematical models of atmospheric diffusion for the study of air pollution by harmful emissions of road transport. It is considered that the specific characteristics of the composition of the exhaust gases, their migration patterns and metabolism in a stratified atmosphere. Maps of atmospheric pollution monitoring are displayed in the example of St. Petersburg ring road.

In the work [10] a mathematical model is constructed to describe the dynamics of one-dimensional nonstationary pollution, as well as from non-stationary sources for a particular specified by the physical state of the atmosphere. The proposed approach uses a direct numerical integration of the exact equations of pollutant transport in the atmosphere with the main physical factors that approximates this method to conduct computational experiment. On the basis of the developed mathematical model, an information system is built for computer modeling of spread of impurities from industrial sources located in the enterprises. The adequacy of the models is verified using the current detection algorithms.

In the work [11], analytical studies of the propagation of emissions from enterprises into atmosphere are conducted. Carbon dioxide (CO2) is considered as the main air pollutant. The paper presents the Green's function for the problem of the occasional instantaneous emissions of harmful impurities in the standard atmospheric boundary layer with a given wind field and an expression is derived for the impurity concentration in the stationary case and in case of continuous source of contamination. Equal levels of air pollution are built and their transformation is analyzed in cases of change of the source parameters.

The problem of modeling the emission of gaseous pollutants in the atmosphere in a new mathematical formulation which allows taking into account the mutual influence of various dynamic processes arising from implementation of the production cycle or as a result of accidents at industrial enterprises is considered in [12]. 
In the work [13], an information system for mathematical modeling of the process of transfer and diffusion of pollutants in the atmosphere with the use of software applications of «ArcGIS», reflecting the real state of the air in places is built. But it should be noted that under this system the results can only be obtained at certain points, and they cannot give an adequate picture of the state of the air in the rest of the territory.

The work [14] is devoted to the development of mathematical models of the dynamics and kinetics of the process of transfer and diffusion of gases and aerosols in the atmosphere. The paper presents a model of the transfer of a multicomponent admixture based on photochemical transformation and formation of aerosols in the troposphere of the northern hemisphere with the account of kinetic processes of enucleation, condensation and coagulation.

A mathematical support of the process of placing inflammable objects and their optimization taking into account the topography and spatial forms, we can see in the work [15].

In the work [16] a computer model is developed to study, forecast and monitor the transport of hazardous substances into the environment from motor vehicles. The numerical implementation of the model on a computer is given using the finite volume method based on a distributed algorithm for computing.

Modeling the field of wind currents on the basis of the Navier-Stokes equations, and taking into account the compressibility of the air environment of turbulence and terrain is proposed in [17], and SIMPLE-algorithm is used as the numerical method of solution.

In [18] studies are carried out on the basis of developed regional models of diffusion of substances, described by hydro-thermodynamic equation, namely the equation of molecular heat conduction in the active layer of soil based on the heat balance of the underlying surface (water, earth). A complex mathematical model, developed by researchers, consists of separate blocks each of which represents a mathematical model that describes the thermodynamic hydro-processes in separate environmental objects. The authors investigated the environmental problems associated with the distribution of pollutants from known sources and determined the probable location of the source in the aquatic environment.

The process of transfer and diffusion of pollutants in the atmosphere, taking into account the different climatic factors and external perturbations are considered in [19]. The paper deals with the transfer of pollutants from the source based on the advection of pollutants from the mean motion of the air, mixing polluting atmospheric turbulence and mass diffusion. In addition, an investigation of the process is presented at various physical and mathematical aspects related to the transport and diffusion of pollutants in the atmospheric boundary layer at the weak and strong winds.

Here, it is necessary to emphasize that the question of considerable interest is in the mathematical modeling of pollutants transported by water. In [20] the problem related to the process of dissemination of harmful substances in the environment is considered and has been modeled as a set of four simple models: onshore flow of water seepage, pollutant transport and deposition of surface runoff pollutants (accumulation) on the surface of the earth. The model is based on the diffusion equation with an additional term on the right side. The developed mathematical model takes into account the influence of topography, lithological structure of territory and the intensity of the contamination rate of absorption of earth surface. The shape, borders and topology of region in the problem changes over time, depending on emergence of dry "islands" surrounded by water.

Work [21] is devoted to the process of dispersion and diffusion of chemically active primary pollutants emitted from elevated linear sources in a stable boundary layer of the atmosphere with a generalized wind speed and a quadratic function of the vertical height. For a given formulation an exact solution is obtained using Laplace transformation for linear sources in the atmospheric boundary layer. Here the chemical reaction that occurs as a result of interaction with the air mass, as well as the conversion of gaseous pollutants in the solid particles and their deposition on the surface of the considered area are taken into account.

In works [22-26], mathematical software is developed for solving the problem of motion of a multicomponent air medium with the account of the transfer and diffusion of pollutants in the atmosphere, changes in the thermal regime of the atmosphere, the phase transition, as well as the influence of vegetation.

The work [27] is devoted to the transport of harmful substances in the air flow in the surface layer of the atmosphere over long and medium distances.

The analysis of abovementioned sources shows that the authors in their studies are not considered the process of transport and diffusion of multicomponent harmful substances into the atmosphere, where a significant role is played by the speed of movement of the air mass of the atmosphere in three directions $u, v$ and $w$, terrain of considered industrial region, heat transfer between the liquid and gaseous phases, the changes in the state density and temperature, etc. which vary during the day and time of year.

It should also be noted that in the mathematical modeling of the spread of harmful substances in the atmosphere, many authors in their works assumed that the spread of harmful substances emitted from sources is 
not considered up to the boundaries of the field of solving the problem and there is no inflow and outflow of harmful substances through them.

In this paper, we study the process of transfer and diffusion of pollutants in the atmosphere by making efforts to fill these gaps.

Based on the foregoing, the purpose of this work is to develop mathematical models and numerical algorithms for solving problems of transfer and diffusion of aerosol emissions in the atmospheric boundary layer.

\section{II.Statement of the problem}

For modeling the process of transfer and diffusion of pollutants in the atmosphere, based on the fundamental laws of hydrodynamics and fluid mechanics of the process we obtain the equations of transportation of pollutants in the atmosphere:

$$
\begin{aligned}
\frac{\partial \theta}{\partial t}+u \frac{\partial h \theta}{\partial x} & +v \frac{\partial h \theta}{\partial y}+\left(w-w_{g}\right) \frac{\partial h \theta}{\partial z}+h \sigma \theta=\frac{\partial}{\partial x}\left(\mu h \frac{\partial \theta}{\partial x}\right)+ \\
& +\frac{\partial}{\partial y}\left(\mu h \frac{\partial \theta}{\partial y}\right)+\frac{\partial}{\partial z}\left(\eta h \frac{\partial \theta}{\partial z}\right)+\delta_{i, j, k} I .
\end{aligned}
$$

Here, we have take into account the velocity of air mass, topography (lay of land), and the diffusion coefficient of turbulence, the speed of precipitation of harmful substances on the surface, the absorption coefficient of pollutants in the atmosphere, and etc.

Considering the state of aggregation of harmful compounds emitted into the atmosphere, we can write the equation describing the transition of water from liquid to gaseous state and vice versa [1-2]

- When gas is emitted from the source

$$
\frac{\partial \theta_{1}}{\partial t}+u \frac{\partial h \theta_{1}}{\partial x}+v \frac{\partial h \theta_{1}}{\partial y}+w \frac{\partial h \theta_{1}}{\partial z}=\frac{\partial}{\partial x}\left(\mu h \frac{\partial \theta_{1}}{\partial x}\right)+\frac{\partial}{\partial y}\left(\mu h \frac{\partial \theta_{1}}{\partial y}\right)+\frac{\partial}{\partial z}\left(\eta h \frac{\partial \theta_{1}}{\partial z}\right)+\delta_{i, j, k} I_{1}
$$

- When the source emits water in gaseous state (water vapor)

$$
\frac{\partial \theta_{2}}{\partial t}+u \frac{\partial h \theta_{2}}{\partial x}+v \frac{\partial h \theta_{2}}{\partial y}+w \frac{\partial h \theta_{2}}{\partial z}=\frac{\partial}{\partial x}\left(\mu h \frac{\partial \theta_{2}}{\partial x}\right)+\frac{\partial}{\partial y}\left(\mu h \frac{\partial \theta_{2}}{\partial y}\right)+\frac{\partial}{\partial z}\left(\eta h \frac{\partial \theta_{2}}{\partial z}\right)+\delta_{i, j, k} I_{2}+\frac{v_{g}}{\rho_{p}}
$$

- when water is supplied from the source

$$
\begin{aligned}
\frac{\partial \theta_{3}}{\partial t}+u \frac{\partial h \theta_{3}}{\partial x}+v \frac{\partial h \theta_{3}}{\partial y}+ & \left(w-w_{g}\right) \frac{\partial h \theta_{3}}{\partial z}+h \sigma_{1} \theta_{3}=\frac{\partial}{\partial x}\left(\mu h \frac{\partial \theta_{3}}{\partial x}\right)+\frac{\partial}{\partial y}\left(\mu h \frac{\partial \theta_{3}}{\partial y}\right)+ \\
& +\frac{\partial}{\partial z}\left(\eta h \frac{\partial \theta_{3}}{\partial z}\right)+\delta_{i, j, k} I_{3}-\frac{v_{g}}{\rho_{p}} ;
\end{aligned}
$$

- and when the source supplies soot

$$
\begin{aligned}
\frac{\partial \theta_{4}}{\partial t}+u \frac{\partial h \theta_{4}}{\partial x} & +v \frac{\partial h \theta_{4}}{\partial y}+\left(w-w_{g}\right) \frac{\partial h \theta_{4}}{\partial z}+h \sigma_{2} \theta_{4}=\frac{\partial}{\partial x}\left(\mu h \frac{\partial \theta_{4}}{\partial x}\right)+ \\
+ & \frac{\partial}{\partial y}\left(\mu h \frac{\partial \theta_{4}}{\partial y}\right)+\frac{\partial}{\partial z}\left(\eta h \frac{\partial \theta_{4}}{\partial z}\right)+\delta_{i, j, k} I_{4} .
\end{aligned}
$$

To determine the concentration of harmful substances in the atmosphere, depending on the orography of the terrain, weather and climatic factors it is necessary to set initial and boundary conditions:

$$
\theta_{i}(x, y, z)=\theta_{i, H}(x, y, z) \text { when } t=0,
$$




$$
\left.\begin{array}{c}
\left.\alpha_{1} \mu \frac{\partial \theta_{i}}{\partial x}\right|_{x=0}=\alpha_{2}\left(\theta_{i}-\theta_{i, H}\right),\left.\beta_{1} \mu \frac{\partial \theta_{i}}{\partial x}\right|_{x=L_{x}}=\beta_{2}\left(\theta_{i}-\theta_{i, H}\right), \\
\left.\left.\alpha_{3} \mu \frac{\partial \theta_{i}}{\partial y}\right|_{y=0}=\alpha_{4}\left(\theta_{i}-\theta_{i, H}\right),\left.\beta_{3} \mu \frac{\partial \theta_{i}}{\partial y}\right|_{y=L_{y}}=\beta_{4}\left(\theta_{i}-\theta_{i, H}\right),\right\} \\
\left.\frac{\partial \theta_{i}}{\partial z}\right|_{z=0}=\xi \theta_{i}-\tilde{f},\left.\frac{\partial \theta_{i}}{\partial z}\right|_{z=L_{z}}=0,
\end{array}\right\}
$$

where $i=1,2,3,4$.

Here $\theta_{1}, \theta_{2}, \theta_{3}, \theta_{4}, \theta_{i, H}$ - respectively the concentrations of pollutants emitted with the water vapor, gas from the source, liquid water, soot and their initial value in the atmosphere; $u, v, w$ - the wind speed in three directions; $v_{g}=f\left(\rho_{n}-\rho_{1}\right)$ - mass evaporation rate; $\rho_{n}$ - the density of the saturated vapor; $\mu$ diffusion coefficient; $\eta$ - the coefficient of turbulence; $\xi$ - the coefficient of the interaction with the underlying ground surface; $h$ - Function describing the orographic surface of the earth; $I_{1}, I_{2}, I_{3}, I_{4}$ - the power of emission sources for harmful gas and water respectively in gaseous form, liquid form, and soot; $\tilde{f}$ - a source of emission of harmful substances from the earth's surface; $\sigma_{1}, \sigma_{2}$ - the coefficients of absorption of harmful substances in the atmosphere (water in liquid form, and soot); $\delta_{i, j, k}$ - Dirac function; $w_{g}$ - The rate of precipitation of harmful particles; $\alpha_{1}, \alpha_{2}, \alpha_{3}, \alpha_{4}, \beta_{1}, \beta_{2}, \beta_{3}, \beta_{4}$ are coefficients (they can take the value of only 0 or 1 depending on the statement of the problem).

From the statement of the problem (1) - (7) it follows that it is necessary to calculate the numerical integration of the velocity of the air mass of the atmosphere in three directions respectively, $u, v$ and $w$.

To determine the velocities of the air mass in the atmosphere in three directions $u, v$ and $w$ we consider the hydrodynamic equations of Navier-Stokes:

$$
\begin{aligned}
& \frac{\partial u}{\partial t}+u \frac{\partial u}{\partial x}+v \frac{\partial u}{\partial y}+w \frac{\partial u}{\partial z}=\frac{1}{\rho} \frac{\partial P}{\partial x}+\frac{\partial}{\partial x}\left(\mu \frac{\partial u}{\partial x}\right)+\frac{\partial}{\partial y}\left(\mu \frac{\partial u}{\partial y}\right)+\frac{\partial}{\partial z}\left(\eta \frac{\partial u}{\partial z}\right)-g_{x}, \\
& \frac{\partial v}{\partial t}+u \frac{\partial v}{\partial x}+v \frac{\partial v}{\partial y}+w \frac{\partial v}{\partial z}=\frac{1}{\rho} \frac{\partial P}{\partial x}+\frac{\partial}{\partial x}\left(\mu \frac{\partial v}{\partial x}\right)+\frac{\partial}{\partial y}\left(\mu \frac{\partial v}{\partial y}\right)+\frac{\partial}{\partial z}\left(\eta \frac{\partial v}{\partial z}\right)-g_{y}, \\
& \frac{\partial w}{\partial t}+u \frac{\partial w}{\partial x}+v \frac{\partial w}{\partial y}+w \frac{\partial w}{\partial z}=\frac{1}{\rho} \frac{\partial P}{\partial x}+\frac{\partial}{\partial x}\left(\mu \frac{\partial w}{\partial x}\right)+\frac{\partial}{\partial y}\left(\mu \frac{\partial w}{\partial y}\right)+\frac{\partial}{\partial z}\left(\eta \frac{\partial w}{\partial z}\right)-g_{z},
\end{aligned}
$$

with the initial and boundary conditions

$$
\left.\begin{array}{c}
\left.u(x, y, z)\right|_{t=0}=\dot{u}_{0}(x, y, z) ;\left.v(x, y, z)\right|_{t=0}=\dot{v}_{0}(x, y, z) ;\left.w(x, y, z)\right|_{t=0}=\dot{w}_{0}(x, y, z), \\
\left.\alpha_{5} \mu \frac{\partial u}{\partial x}\right|_{x=0}=\alpha_{6}\left(u-\dot{u}_{0}\right),\left.\beta_{5} \mu \frac{\partial u}{\partial x}\right|_{x=L_{x}}=\alpha_{6}\left(u-\dot{u}_{0}\right), \\
\left.\alpha_{7} \mu \frac{\partial v}{\partial y}\right|_{y=0}=\alpha_{8}\left(v-\dot{v}_{0}\right),\left.\beta_{7} \mu \frac{\partial v}{\partial y}\right|_{y=L_{y}}=\alpha_{8}\left(v-\dot{v}_{0}\right), \\
\left.\eta \frac{\partial w}{\partial z}\right|_{z=0}=0,\left.\eta \frac{\partial w}{\partial z}\right|_{x=L_{z}}=0 .
\end{array}\right\}
$$


Here, $P$ - pressure; $g$ - acceleration of free fall.

To calculate the density of the substances emitted into the atmosphere, taking into consideration the conservation of mass in the fluid flow through the fixed volume we obtain continuity equation

$$
\frac{\partial \rho}{\partial t}+\frac{\partial(\rho u)}{\partial x}+\frac{\partial(\rho v)}{\partial y}+\frac{\partial(\rho w)}{\partial z}=\frac{\partial}{\partial x}\left(\mu \frac{\partial \rho}{\partial x}\right)+\frac{\partial}{\partial y}\left(\mu \frac{\partial \rho}{\partial y}\right)+\frac{\partial}{\partial z}\left(k \frac{\partial \rho}{\partial z}\right)+I_{g}
$$

with the appropriate initial and boundary conditions:

$$
\begin{gathered}
\left.\rho(x, y, z)\right|_{t=0}=\rho_{c} \\
\left.\alpha_{9} \rho \mu \frac{\partial \rho}{\partial x}\right|_{x=0}=\alpha_{10}\left(\rho-\rho_{0}\right),\left.\beta_{9} \rho \mu \frac{\partial \rho}{\partial x}\right|_{x=L_{x}}=\beta_{10}\left(\rho-\rho_{0}\right), \\
\left.\alpha_{11} \rho \mu \frac{\partial \rho}{\partial y}\right|_{y=0}=\alpha_{12}\left(\rho-\rho_{0}\right),\left.\beta_{11} \rho \mu \frac{\partial \rho}{\partial y}\right|_{y=L_{y}}=\beta_{12}\left(\rho-\rho_{0}\right), \\
\left.\frac{\partial \rho}{\partial z}\right|_{z=0}=0,\left.\frac{\partial \rho}{\partial z}\right|_{z=L_{z}}=0 .
\end{gathered}
$$

Since contaminants emitted into the environment have a certain temperature, which plays a significant role in the propagation of harmful substances in the atmosphere, the account of this factor is necessary. The equation describing the exchange processes and diffusion of heat and heat transfer into the environment is as follows:

$$
\begin{aligned}
\frac{\partial \Phi}{\partial t}+u \frac{\partial \Phi}{\partial x} & +v \frac{\partial \Phi}{\partial y}-w_{g} \frac{\partial \Phi}{\partial z}=\frac{\partial}{\partial x}\left(\mu \frac{\partial \Phi}{\partial x}\right)+\frac{\partial}{\partial y}\left(\mu \frac{\partial \Phi}{\partial y}\right)+\frac{\partial}{\partial z}\left(\mu \frac{\partial \Phi}{\partial z}\right)+ \\
& +\frac{\partial}{\partial x}\left(\lambda \frac{\partial T}{\partial x}\right)+\frac{\partial}{\partial y}\left(\lambda \frac{\partial T}{\partial y}\right)+\frac{\partial}{\partial z}\left(\lambda \frac{\partial T}{\partial z}\right)+\delta_{i, j, k} I_{T} .
\end{aligned}
$$

Here $\Phi$ - the thermal energy; $w_{g}$ - the rate of precipitation of suspended particles; $\lambda$ - coefficient of thermal conductivity; $I_{T}$ - the function describing the distribution and power of heat source.

Since we consider the process of distributing multi-component environment, then for the coefficient of thermal conductivity and thermal energy the following relation is true

$$
\Phi=\sum_{i=1}^{L} \Phi_{i} \theta_{i}=\sum_{i=1}^{L} \rho_{i} c_{i} \theta_{i} T_{i}
$$

In our problem statement of heat transfer in a multicomponent environment we can consider the cases:

- Heat transfer for gas

$$
R_{1} \frac{\partial T}{\partial t}=\frac{\partial}{\partial x}\left(R_{2} \frac{\partial T}{\partial x}\right)+\frac{\partial}{\partial y}\left(R_{2} \frac{\partial T}{\partial y}\right)+\frac{\partial}{\partial z}\left(R_{2} \frac{\partial T}{\partial z}\right)+\bar{q}_{2} v_{g}-\alpha_{v}\left(T-T_{s}\right),
$$

where $R_{1}=\sum_{i=1}^{2} \rho_{i} c_{\rho} \theta_{i} ; R_{2}=\sum_{i=1}^{2}\left(\rho_{i} c_{\rho} \mu+\lambda_{i}\right) \theta_{i}$; - mass evaporation rate; $\rho, c_{\rho}$ - the density and specific heat of gas phase; $\bar{q}$ - specific heat of transformation, $T, T_{s}$ - the temperature of the gas and condensed phases; $\alpha_{v}$ - Heat transfer coefficient;

- Heat transfer for the condensate 


$$
R_{3}\left(\frac{\partial T}{\partial t}-w_{0} \frac{\partial T}{\partial z}\right)=\frac{\partial}{\partial x}\left(\left(R_{3} \mu\right) \frac{\partial T_{s}}{\partial x}\right)+\frac{\partial}{\partial y}\left(\left(R_{3} \mu\right) \frac{\partial T_{s}}{\partial y}\right)+\frac{\partial}{\partial z}\left(\left(R_{3} \mu\right) \frac{\partial T_{s}}{\partial z}\right)-\alpha_{v}\left(T-T_{s}\right)
$$

where $R_{3}=\sum_{i=3}^{4}\left(\rho_{i} c_{\rho_{i}} \theta_{i}\right) ; c_{\rho_{i}}, \theta_{i}, \rho_{i}$ - the specific heat capacity, the volume fractions of $i$-th phase and the real densities respectively; $T, T_{s}$ - The temperature of the gas and condensed phases.

To solve (16) - (17) we give the initial and boundary conditions:

$$
\begin{gathered}
T(x, y, z)=T_{0}(x, y, z) ; \\
\left.\alpha_{13} k_{1} \frac{\partial T}{\partial x}\right|_{x=0}=\alpha_{14}\left(T-T_{0}\right),\left.\quad \beta_{13} k_{1} \frac{\partial T}{\partial x}\right|_{x=L_{x}}=\beta_{14}\left(T-T_{0}\right), \\
\left.\alpha_{15} k_{1} \frac{\partial T}{\partial y}\right|_{y=0}=\alpha_{16}\left(T-T_{0}\right),\left.\quad \beta_{15} k_{1} \frac{\partial T}{\partial y}\right|_{y=L_{y}}=\beta_{16}\left(T-T_{0}\right), \\
\left.\alpha_{17} k_{1} \frac{\partial T}{\partial z}\right|_{z=0}=\alpha_{18}\left(T-T_{0}\right),\left.\quad \beta_{17} \mathrm{k}_{1} \frac{\partial T}{\partial z}\right|_{z=L_{z}}=\beta_{18}\left(T-T_{0}\right) .
\end{gathered}
$$

To determine the vapor pressure in terms of temperature, we use the equation of Mendeleev-Clapeyron, and by differentiating it we obtain

$$
\frac{\rho}{P} \frac{\partial P}{\partial t}=\frac{\partial \rho}{\partial t}+\frac{\rho}{T} \frac{\partial T}{\partial t}
$$

Here $\rho_{i}, R, M$ - the density, the universal gas constant, the molar mass; $k_{1}$ - coefficient of thermal conductivity.

In equations (2) - (19), depending on the statement of problem, the values of $\alpha_{1}, \alpha_{2}, \alpha_{3}, \alpha_{4}, \beta_{1}, \beta_{2}, \beta_{3}, \beta_{4}, \alpha_{5}, \alpha_{6}, \alpha_{7}, \alpha_{8}, \beta_{5}, \beta_{6}, \beta_{7}, \beta_{8} \alpha_{9}, \alpha_{10}, \alpha_{11}, \alpha_{12}, \beta_{9}, \beta_{10}, \beta_{11}, \beta_{12}$ $\alpha_{13}, \alpha_{13}, \alpha_{14}, \alpha_{15}, \beta_{13}, \beta_{14}, \beta_{15}, \beta_{16}, \alpha_{16}, \alpha_{17}, \alpha_{18}, \beta_{16}, \beta_{17}, \beta_{18}$ can be only 0 or 1 .

To solve the equations (8) - (12) we split them into physical parameters and get two problems, the first of which is to be written in the difference analogue:

$$
\begin{aligned}
& \frac{u^{n+0.5}-u^{n}}{\Delta t / 2}+u \frac{\partial u}{\partial x}+v \frac{\partial u}{\partial y}+w \frac{\partial u}{\partial z}=\frac{\partial}{\partial x}\left(\mu \frac{\partial u}{\partial x}\right)+\frac{\partial}{\partial y}\left(\mu \frac{\partial u}{\partial y}\right)+\frac{\partial}{\partial z}\left(\mu \frac{\partial u}{\partial z}\right)-G_{x}, \\
& \frac{v^{n+0.5}-v^{n}}{\Delta t / 2}+u \frac{\partial v}{\partial x}+v \frac{\partial v}{\partial y}+w \frac{\partial v}{\partial z}=\frac{\partial}{\partial x}\left(\mu \frac{\partial v}{\partial x}\right)+\frac{\partial}{\partial y}\left(\mu \frac{\partial v}{\partial y}\right)+\frac{\partial}{\partial z}\left(\mu \frac{\partial v}{\partial z}\right)-G_{y}, \\
& \frac{w^{n+0.5}-w^{n}}{\Delta t / 2}+u \frac{\partial w}{\partial x}+v \frac{\partial w}{\partial y}+w \frac{\partial w}{\partial z}=\frac{\partial}{\partial x}\left(\mu \frac{\partial w}{\partial x}\right)+\frac{\partial}{\partial y}\left(\mu \frac{\partial w}{\partial y}\right)+\frac{\partial}{\partial z}\left(\mu \frac{\partial w}{\partial z}\right)-G_{z},
\end{aligned}
$$

and the second

$$
\begin{gathered}
\frac{u^{n+1}-u^{n+0.5}}{\Delta t / 2}=-\frac{1}{\rho} \frac{\partial P}{\partial x}, \\
\frac{v^{n+1}-v^{n+0.5}}{\Delta t / 2}=-\frac{1}{\rho} \frac{\partial P}{\partial y}, \\
\frac{w^{n+1}-w^{n+0.5}}{\Delta t / 2}=-\frac{1}{\rho} \frac{\partial P}{\partial z} .
\end{gathered}
$$


Then, by multiplying the system of equations (20) to $\rho \Delta t / 2$ and by differentiating respect to variables $x, y, z$ and as a result we get

$$
\left.\begin{array}{l}
\left(\rho \frac{\partial u}{\partial x}\right)^{n+1}=\left(\rho \frac{\partial u}{\partial x}\right)^{n+0,5}-\Delta t / 2 \frac{\partial^{2} P}{\partial x^{2}}, \\
\left(\rho \frac{\partial v}{\partial y}\right)^{n+1}=\left(\rho \frac{\partial v}{\partial y}\right)^{n+0,5}-\Delta t / 2 \frac{\partial^{2} P}{\partial y^{2}}, \\
\left(\rho \frac{\partial w}{\partial z}\right)^{n+1}=\left(\rho \frac{\partial w}{\partial z}\right)^{n+0,5}-\Delta t / 2 \frac{\partial^{2} P}{\partial z^{2}} .
\end{array}\right\}
$$

Substituting the system (21) into (13) we obtain the following:

$$
\begin{gathered}
\frac{\partial \rho}{\partial t}+\left(\frac{\partial(\rho u)}{\partial x}\right)^{n+0.5}-\Delta t / 2 \frac{\partial^{2} P}{\partial x^{2}}+\left(\frac{\partial(\rho v)}{\partial y}\right)^{n+0.5}- \\
\left.-\Delta t / 2 \frac{\partial^{2} P}{\partial y^{2}}+\left(\frac{\partial(\rho w)}{\partial z}\right)\right)^{n+0.5}-\Delta t / 2 \frac{\partial^{2} P}{\partial z^{2}}= \\
=\left(\mu \frac{\partial^{2} \rho}{\partial x^{2}}\right)+\left(\mu \frac{\partial^{2} \rho}{\partial y^{2}}\right)+\left(\mu \frac{\partial^{2} \rho}{\partial z^{2}}\right)+I_{p}
\end{gathered}
$$

Using the equation of state (19), we obtain an equation for calculating the pressure field:

$$
\begin{aligned}
\left(\frac{\rho}{P} \frac{\partial P}{\partial t}\right)=\Delta t & / 2 \frac{\partial^{2} P}{\partial x^{2}}+\Delta t / 2 \frac{\partial^{2} P}{\partial y^{2}}+\Delta t / 2 \frac{\partial^{2} P}{\partial z^{2}}-\frac{\rho}{T} \frac{\partial T}{\partial t}- \\
-\left(\rho \frac{\partial u}{\partial x}\right)^{n+0,5} & -\left(\rho \frac{\partial u}{\partial y}\right)^{n+0,5}-\left(\rho \frac{\partial w}{\partial z}\right)^{n+0,5}+\left(\mu \frac{\partial^{2} \rho}{\partial x^{2}}\right)+ \\
& +\left(\mu \frac{\partial^{2} \rho}{\partial y^{2}}\right)+\left(k \frac{\partial^{2} \rho}{\partial z^{2}}\right)+I_{g} .
\end{aligned}
$$

With the resulting equation (23) we can calculate the field of pressure distribution in considered layer of the atmosphere.

Thus, a three-dimensional mathematical model of spread of harmful substances in the atmospheric boundary layer is developed, taking into account the terrain and the characteristics of the underlying surface. In the development of this model we use: the equation of motion of a multicomponent air, pressure calculation model, the model of heat flow, which is described by the thermal conductivity of gas and condensate. Using the given model we can calculate the main indicators and parameters that affect the process of transfer and diffusion of harmful multicomponent mixtures emitted from industrial facilities, construction and industrial sites and drained parts of seas and lakes.

\section{Conclusion}


A mathematical models of transport and diffusion of pollutants in the form of water, gas and soot in a multicomponent air is developed, taking into account the factors such as the transition of water from liquid to gaseous state, turbulent exchange, convective motion, the precipitation of substances, heat transfer between the liquid and gaseous states, density and temperature changes, as well as consideration of the terrain that greatly affects the dynamically changing state of the studied object.

In the development of a mathematical model of the spread of harmful substances in the atmosphere we have derived an equation for calculating the pressure field, which takes into account the compressibility of the medium, the thermal expansion, the turbulent mixing of the air mass of the atmosphere.

The peculiarity of the developed mathematical model of transport and diffusion of pollutants in the atmospheric boundary layer and air traffic environment is related to the integration of turbulent mixing in the equation of continuity of environment, as well as the consideration of the effect of orographic surface of the ground vegetation on the distribution of aerosol particles in the atmosphere.

In the developed mathematical model, the transfer and diffusion of harmful components are taken into account through the boundaries of solution area of the problem, using the boundary condition of the third type, which corresponds to the actual physical nature of the process.

Taking into account the above factors, the developed mathematical model describes the process more adequately compared to known models offered by other authors.

\section{References:}

[1] Pavel PV Mathematical modeling of non-stationary turbulent diffusion using the finite element method // Materials of III region. Conf. "University science - the North Caucasus region." - Stavropol: NCSTU, 1999. - S. 7.

[2] PV Korchagin Building a computational scheme for the transport equation using the method of weighted residuals and the finite element method // All-Russia. scientific. Conf. "Mathematical modeling in scientific research." - Stavropol: SGU, 2000. - P. 55-58.

[3] PV Korchagin Modeling joint dissemination reactants // Proceedings of the III Mezhregion. Conf. "Students' Science - the Russian economy." - Stavropol: NCSTU, 2002, pp 4-5.

[4] Lisanov MV Pchelnikov A., Sumy SI Dispersion modeling of emissions of hazardous substances in the atmosphere of the Society Ros.him.zhurnal them. DI. Medeleeva t.HLIX 2005, number 4, Article 18-28

[5] Belihov AB, Legotin DL, Sukhov AK Modern computer models of the spread of pollutants in the atmosphere Gazette Sh. on. Nekrasov 14. № 1, 2013 science

[6] Berlyand ME Modern problems of atmospheric diffusion and air pollution. - L .: Gidrometeoizdat, 1975. - 448

[7] Volkov VY, Abbas SB The automated system of support for research dissemination of pollutant emissions in the atmosphere News Tula State University. Engineering Issue number 2/2013

[8] Lozhkyn VN, VV Medeyko Models of environmental damage assessment, applied in the Russian Federation, the United States and the EU, with the state regulation of the impact of vehicles on the environment // Information byulleten.№ 2 (32). "The issues of air pollution." SPb .: SPC "atmosphere" at the MGO them. AI Voeikov, 2005, pp 103-116.

[9] Uliasz M., Stocker R.A., Pielke R.A. Regional modelling of air pollution transport in the south-western USA. (In :) Zannetti P. (ed.), Environmental Modelling Vol. III Comput. Mech. Public. Southampton, 1996. 34 pp.

[10] Belosludtsev AA Gusarov DV, Eremin, MA, NM Kuzmin, AV Khoperskaya snoring SS Information and computer system for modeling the dynamics of impurities from the chemical industry Volgograd Bulletin State University. Series 1: Mathematics. Physics Vypusk№ 12/2009, p. 24-31

[11] Chernyavskiy S Mathematical model of process of distribution of gas pollutants in

the atmosph ere under different weather conditions XX International correspondence scientific-practical conference "Engineering - From Theory to Practice" (Novosibirsk, Russia, April 17, 2013). from. 17-22

[12] Braces YA Mathematical modeling of emissions and atmospheric dispersion of gaseous impurities Bulletin of Kharkov National University. Series "Matematiseskoe modeling. Automation control systems »№ 775 ., 2007., p. 236-245.

[13] Smirnov EA Information system for the modeling of air pollution using ArcGIS // Topical Issues Technical Science: Proceedings of the international. scientific. Conf. - Perm, 2011. - P. 27-31.

[14] Aloyan AE The dynamics and kinetics of trace gases and aerosols in the atmosphere. - M .: INM RAS, 2002. $-201 \mathrm{p}$.

[14] Chub AI The mathematical model of optimization problem placing inflammable objects based on topography layout area // Radioelektronika, informatika, upravlinnya release. - 2013. - № 1. - S. 88-93. 
[15] Sukhinov AI, Gadelshin VK Lyubomischenko DS Mathematical model of the spread of harmful emissions from vehicles, based on control volume method and its parallel implementation on a distributed computing cluster // Southern Federal University. Technical science. - 2009. - № 2. - Volume 91. - C. 8-14

[16] Gadelshin VK Lyubomischenko DS Sukhinov AI Mathematical modeling of a field of wind currents and distribution of contaminants in urban terrain based k- $\varepsilon$-turbulence model // Southern Federal University. Technical science. - 2010. - № 6. - Volume 107. - C. 48-67.

[17] Kordzadze A. Mathematical modelling of dynamical and ecological processes in the system sea-landatmosphere // Air, Water and Soil Quality Modelling for Risk and Impact Assessment. - 2007. - PP. 181193.

[18] Sharan M., Gopalakrishnan S.G. Mathematical modeling of diffusion and transport of pollutants in the atmospheric boundary layer // January pure and applied geophysics. - 2003. - Vol. 160. - Issue 1-2. - PP. 357-394.

[19] Gitis VG, Petrova EN, Pirogov SA, Yurkov EF Mathematical modeling of the pollutants overland flow and transport // Automation and Remote Control. - 2007. - Vol. 68. - Issue 9. - PP. 1643-1653.

[20] Khan Y., Shekhu M., Sulochana C. Mathematical model for dispersion and diffusion of chemically reactive pollutants from various sources into a boundary layer with dry deposition // Engineering Computations. 2013. - Vol. 30. - Issue 5. - PP. 707 - 727.

[21] Sukhinov AI, AE Chistyakov, Hachun DS Mathematical modeling of motion of a multicomponent air and transport of pollutants // Southern Federal University. Technical science. - 2011. - № 8. - S. 73-79.

[22] AE Chistyakov, Hachunts DS The problem of motion of a multicomponent air quality in view of vaporization and condensation // Southern Federal University. Technical science. - 2013. - № 4. - pp 87-98.

[23] Sukhinov AI DS Hachunts Software implementation of the two-dimensional problem of traffic air pollution // Southern Federal University. Technical science. - 2013. - № 4. - pp 15-20.

[24] AE Chistyakov The three-dimensional model of the motion of water environment in the Sea of Azov taking into account transport of salt and heat // Southern Federal University. Technical science. - 2009. - № 8. - S. 75-82.

[25] Ravshanov N., Shertaev M., Toshtemirova N. Mathematical Model for the Study and Forecast of the Concentration of Harmful Substances in the Atmosphere // American Journal of Modeling and Optimization. - 2015. - Vol. 3. - № 2. - PP. 35-39.

[26] Ravshanov N. Sharipov DK, Ahmedov D. Modeling of pollution, taking into account the terrain climatic factors // Information Technology modeling and management - Voronezh, 2015.№3. - S. 222-235.

[27] Anderson G.E. Musicales influences on wind fields J. Appl. Meteor., 1971,10, pp. 377-386. 